\title{
Feierliche Wiedereröffnung des Palmengarten-Gesellschaftshauses
}

\author{
Hilke Steinecke
}

Zusammen mit dem angrenzenden Palmenhaus ist das Gesellschaftshaus das historische Herzstück des 1871 eröffneten Palmengartens. Heinrich Siesmayer, Gründer des Palmengartens, träumte von einem „Südpalast“, einem öffentlichen Wintergarten mit angrenzender Restauration. Nach Vorschlag des ersten Garteninspektors Heiss wurde die Einrichtung Palmengarten genannt. In der Mitte des 19. Jahrhunderts entwickelte sich der Stil des Eklektizismus und wurden Gebäude-Ensembles aus Glashaus und Repräsentationsarchitektur modern. Auch das Gesellschaftshaus wurde in diesem Stil errichtet. Zusammen mit dem Palmenhaus hat es den Charakter eines Bahnhofsgebäudes mit der Bahnhofshalle. Das Filetstück des Gesellschaftshauses ist der Festsaal, der ursprünglich prunkvoll mit Stuck, Wandgemäl- den, repräsentativen Kronleuchtern und Motiven aus der Antike (z.B. zwei Karyatiden um einen Fensterbogen; das sind in der Architektur verwendete weibliche Figuren mit Stützfunktion) ausgestattet war.

Die Geschichte des Gesellschaftshauses ist vielschichtig und reicht von der Zerstörung durch einen Brand bereits 10 Jahre nach der Palmengarten-Gründung über verschiedene Veränderungen wie die gravierenden Umbauten 1928/29 im sachlich-nüchternen Bauhausstil unter Martin Elsässer. Im zweiten Weltkrieg gab es Beschädigungen durch Bombenangriffe und es folgte eine kurze Zeit der Nutzung durch die Amerikaner. Stuck und Schmuck im Festsaal galten ab den 1950er Jahren nicht mehr als zeitgemäß und waren z.T. im schlechten Zustand, sodass die Reste des ehemaligen Prunks

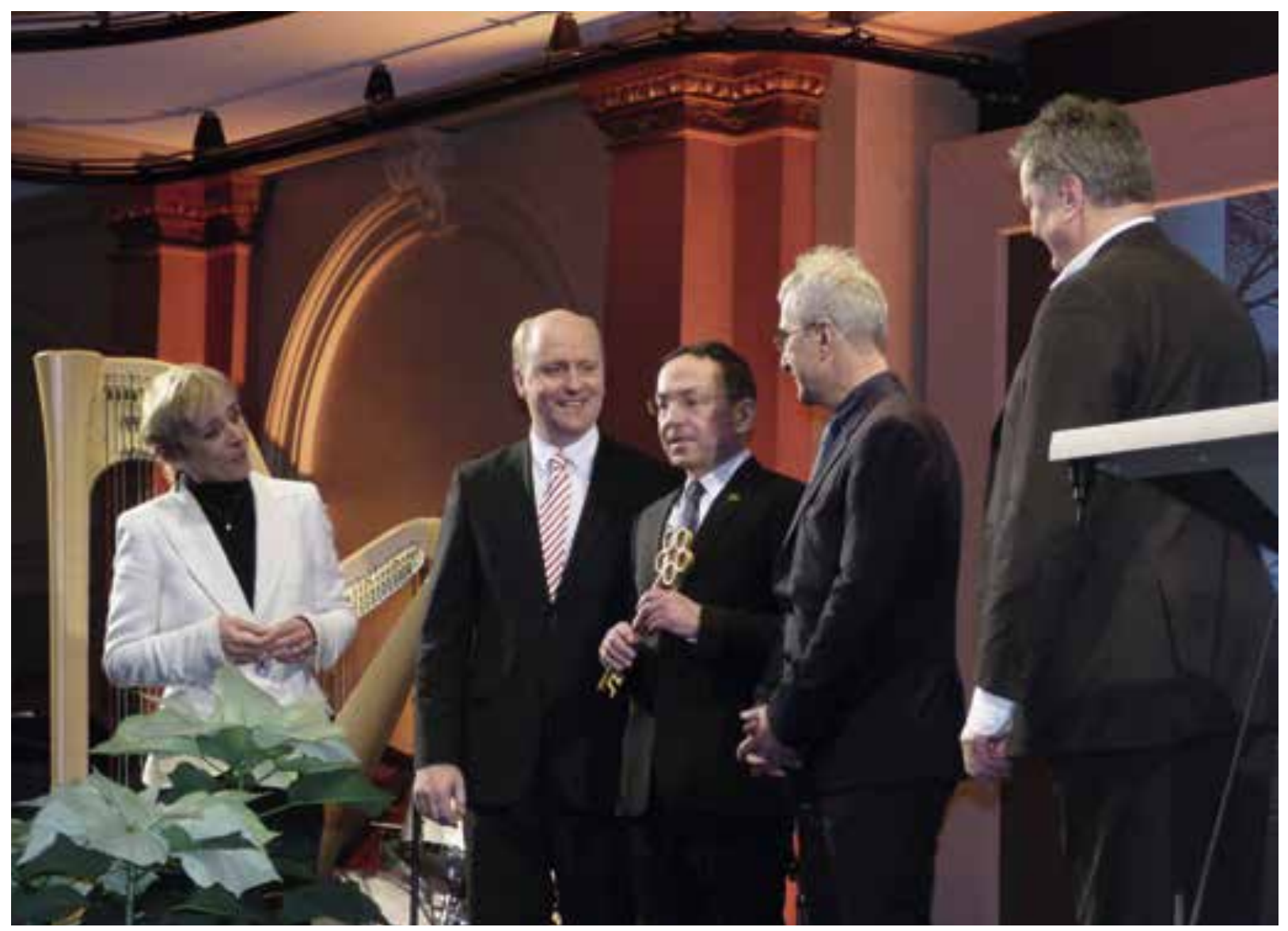


hinter Wandverkleidungen versteckt wurden. Über die Geschichte des Gesellschaftshauses von den Anfängen bis heute berichtet ausführlich SAbIne Börchers in ihrem 2012 pünktlich zur Wiedereröffnung erschienenen Buch.

Bereits der ehemalige Palmengarten-Direktor Gustav Schoser hatte die Vision von einer umfangreichen Renovierung des Gesellschaftshauses und einer Wiederherstellung der früheren Schönheit des Festsaales. Immerhin wurde der Festsaal im nach dem Brand errichteten Neubau von Friedrich von Thiersch geplant. Der Architekt und Maler gilt als einer der wichtigsten Vertreter des Späthistorismus in der Gründer- und wilhelminischen Zeit. Auf ihn geht auch das Wiesbadener Kurhaus zurück.

Das Renovierungsvorhaben scheiterte zunächst aus finanziellen Gründen. Erst nach der Jahrtausendwende wurde eine Totalsanierung spruchreif. Aufgrund von erheblichen Baumängeln wurde 2002 das Gesellschaftshaus zunächst geschlossen, der Pachtvertrag mit dem bisherigen Betreiber der Gastronomie lief aus. Eigentlich sollte der Umbau bereits 2004 abgeschlossen sein, doch verzögerte sich aus verschiedenen Gründen der Baubeginn erheblich. Bis 2009 fiel das Gesellschaftshaus regelrecht in einen Dornröschenschlaf, bis es schließlich erst 2009 mit den Bauarbeiten losging. Den Architektenwettbewerb zur Sanierung des Gesellschaftshauses hatte zuvor der Stararchitekt David Chipperfield gewonnen.

Von vielen wurden die baulichen Fortschritte aufmerksam verfolgt, aber auch die damit verbundenen unausweichlichen Baumfäll-Aktionen kritisch betrachtet. So mussten u.a. die unter Gustav Schoser vor der Terrasse gepflanzten und mittlerweile recht stattlichen Pinien weichen. Vier große Chinesische Hanfpalmen links und rechts des Hauses wurden ausgegraben und vor das Historische Eingangsschauhaus an der Siesmayerstraße gepflanzt, um die dort bereits bestehende Palmengruppe

Abb. 1 (Seite 72): Feierliche Schlüsselübergabe.

Abb. 2: Eintrag in das Goldene Stiftungsbuch der Stadt Frankfurt.

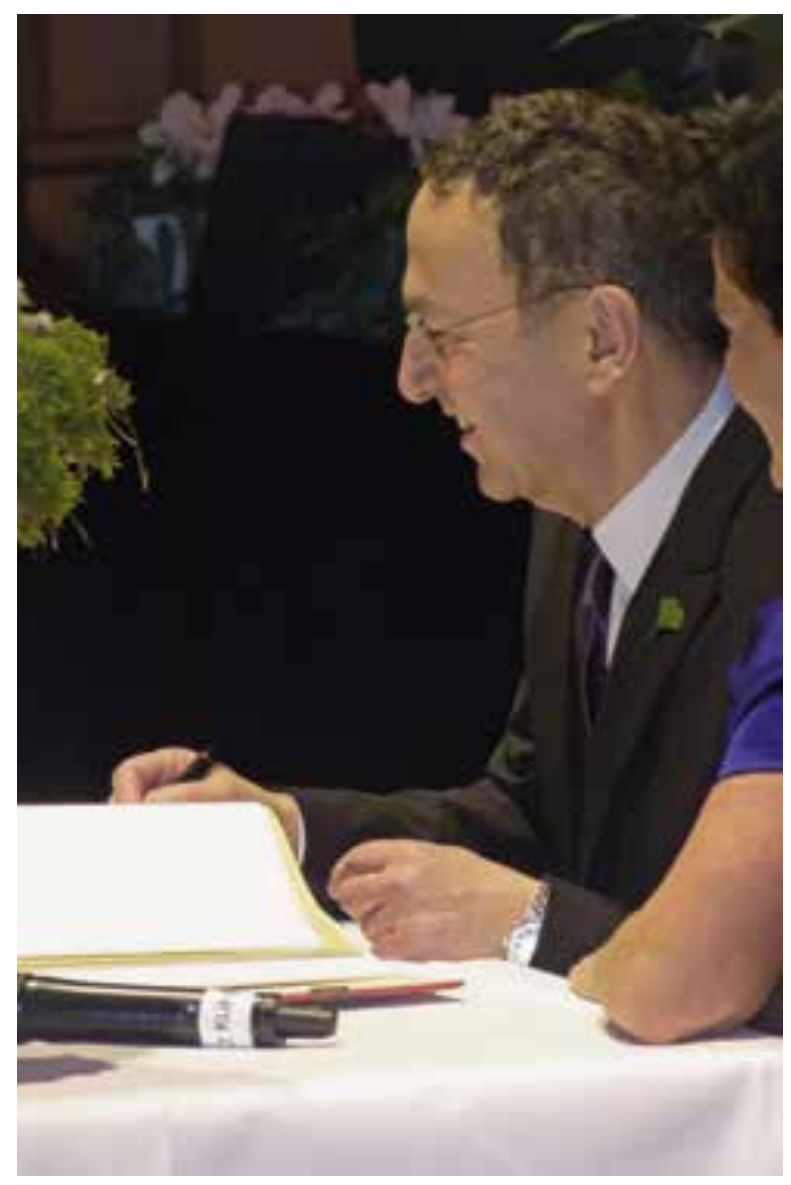

zu erweitern. Wie es oft bei so diffizilen Bauprojekten vorkommt, gibt es unvorhergesehene Schwierigkeiten, verlängert sich die Bauzeit und sind die Kosten viel höher als zunächst angenommen. Insgesamt hat die Sanierung rund 40 Mio. Euro gekostet. Im Herbst 2012 war es dann endlich soweit, dass erste Veranstaltungen stattfinden konnten. Der Festsaal hat eine Kapazität für 1000 Personen.

Das Gesellschaftshaus mit seiner GourmetGastronomie wird nun vom Dreierteam RoBERT Mangold (Geschäftsführer), Margareta Dillinger und Johnny Klinke (Gesellschafter) betrieben.

Am Abend des 8. Dezembers 2012 wurde die Wiedereröffnung des Festsaales mit einem Festakt begangen. An einem strahlenden Wintertag mit Eis und Schnee fanden sich gegen 19.30 Uhr rund 500 geladene Gäste ein, z.T. Vertreter aus der Stadtverwaltung, der Kunst- und Kulturszene sowie aus dem öffentlichen Leben Frankfurts. Der Oberbürgermeister Peter FELdmann war leider verhindert, in Vertretung 


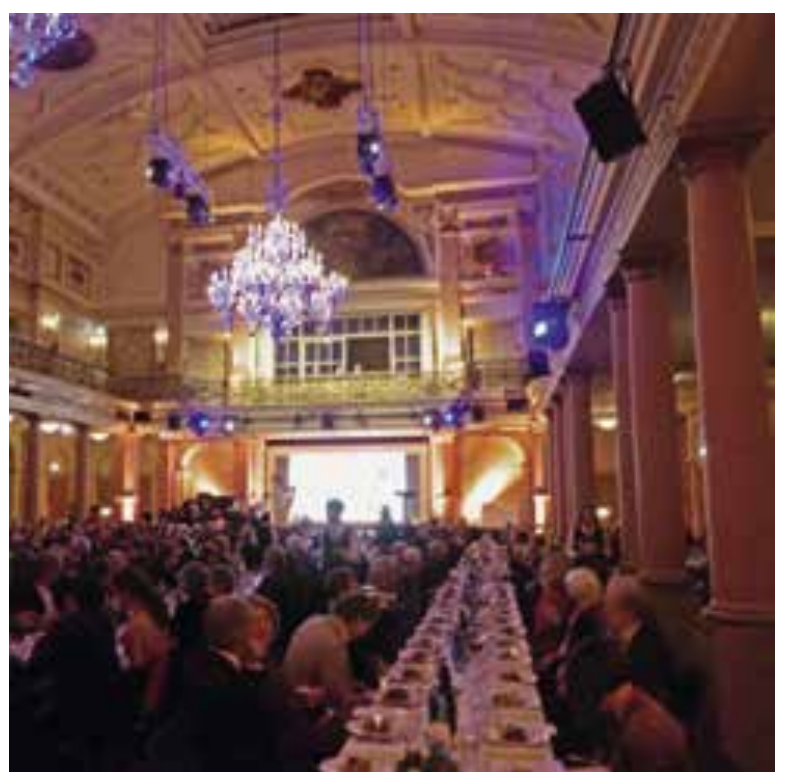

hielt Stadtkämmerer Uwe BECKER eine Begrüßungsrede. Anschließend überreichte er ein Geschenkpaket - das Gesellschaftshaus symbolisierend - an Matthias Jenny, den Leiter des Palmengartens. Dieser freute sich sehr über den überdimensional großen goldenen Schlüssel, der extra für den Festakt in der PalmengartenSchlosserei angefertigt worden war. Nicht lange blieb er in Jennys Händen, denn er reichte ihn an Robert Mangold weiter. Auch Prinzessin von Hannover, Vorsitzende der Palmengarten-Gesellschaft, richtetet ein Grußwort an die Anwesenden. Wichtiger Programmpunkt war anschließend der Eintrag der Stiftung Palmengarten und Botanischer Garten in das Goldene Buch der Frankfurter Stiftungen durch Matthias Jenny und die Umweltdezernentin Rosemarie Heilig.

Die Gartenschriftstellerin Eva Demski hielt den Festvortrag „Pracht für alle“ mit Bezug auf den Festsaal, der ursprünglich für edle Feierlichkeiten des gehobenen Frankfurter Bürgertums vorgesehen war. Zwischendurch gab es verschiedene musikalische Einlagen von Studierenden der Hochschule für Musik und Darstellende Kunst, darunter auch Harfe und Gesang. Die HR Bigband spielte schwungvolle Ohrwürmer. Wie in alten Zeiten wurde das Publikum aufgefordert, zur swingenden Musik das Tanzbein zu schwingen. Auch Matthias Jenny und Rosemarie Heilig nutzten die Ge-

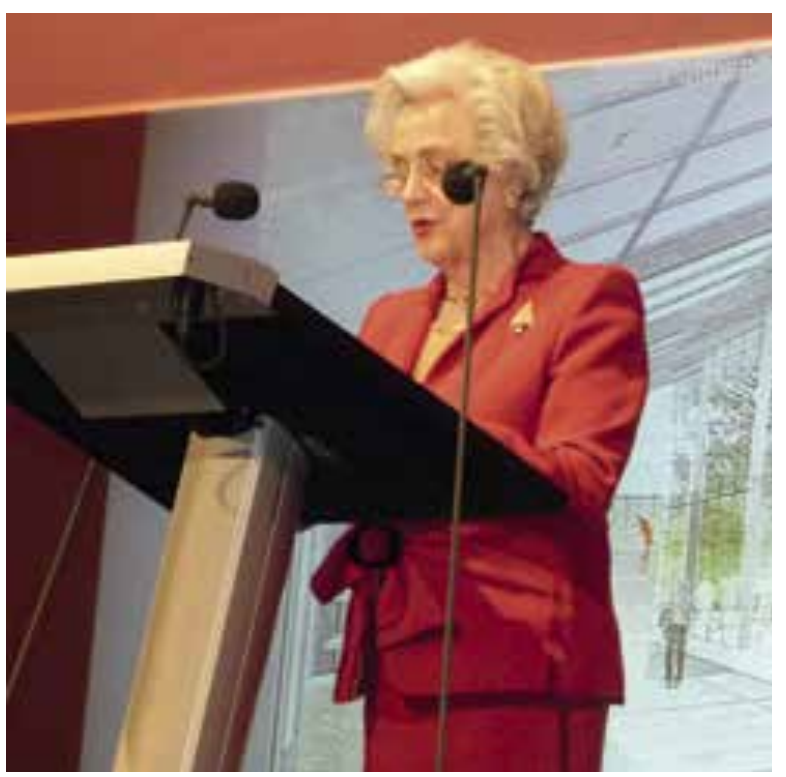

legenheit für einen gemeinsamen Walzer. Manch einer der etwas älteren Generation erinnerte sich gern an die beliebten Tanzbälle im Festsaal; und so manche Liebe nahm ihren Anfang im Palmengarten.

Für das leibliche Wohl sorgte die Tiger und Palmen GmbH. Passend zum Ambiente im schönsten Saal Frankfurts gab es im Menü auch die Frankfurter Traditionspezialität „Kartoffeln mit Frankfurter Grüner Soße“. Der im Tigerpalast regelmäßig auftretende russische Akrobat Oleg Izossimov gab eine kleine Kostprobe von seinem artistischen Können. Für eine feierliche Stimmung sorgte zudem die Beleuchtung des Saales mit rotem, blauem und violettem Licht. Interessante Beleuchtungseffekte ergaben sich auch auf den nach historischen Vorbildern nachempfundenen Kronleuchtern aus dem alten Festsaal. Ein besonderer Reiz des Festsaales besteht darin, von ihm aus direkt in das Palmenhaus zu gelangen und dort dann ein tropisches Ambiente unter Palmen genießen zu können. Im Palmen-

Abb. 3 (links): Festliche Atmosphäre im gefüllten Saal. Abb. 4 (rechts): Grußworte der Prinzessin von Hannover. 


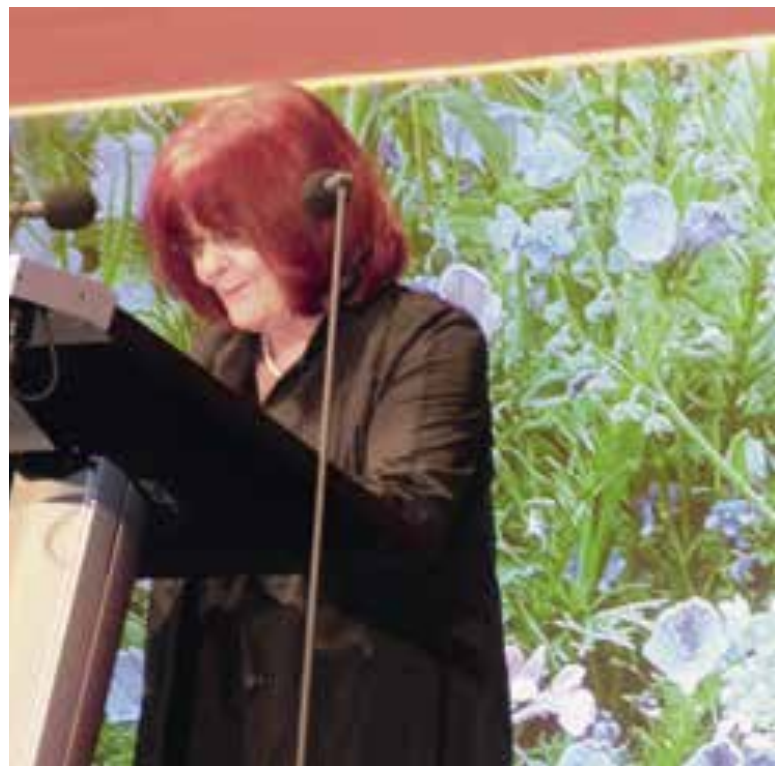

haus herrschte eine ganz besondere Atmosphäre, denn im Rahmen der gerade eröffneten Winterlicht-Veranstaltung gab es im ganzen Park, darunter auch im Palmenhaus, geheimnisvoll illuminierte Bereiche.

Während des ganzen Abends wurden auf der Bühne großformatige Bilder aus dem Palmengarten und dem Botanischen Garten aus allen Jahreszeiten projiziert, um die Schönheit und Vielfalt der Gärten zu vergegenwärtigen.

Der festliche Abend war sehr gelungen und wurde auch von den Medien mit großem Interesse aufgenommen. Spannend bleibt es noch, inwieweit der Festsaal nun wirklich auch von Frankfurter Bürgern genutzt werden kann oder überwiegend nur für zahlungskräftige Veranstalter geschlossener Gesellschaften zur Verfügung steht. Fest steht, dass er auf jeden Fall während der Gartenmesse und des Rosen- und Lichterfestes für alle geöffnet sein wird. Ein neues Kapitel in der spannenden Geschichte des Gesellschaftshauses und des schönsten Saales Frankfurts ist aufgeschlagen.

Abb. 5 (links): Eva Demski hält den Festvortrag.

Abb. 6 (rechts): Eine der Musiker-Büsten im Festsaal.

Abb. 7 (unten): Karyatide an einem Fenster im Festsaal mit Blick auf das Palmenhaus.
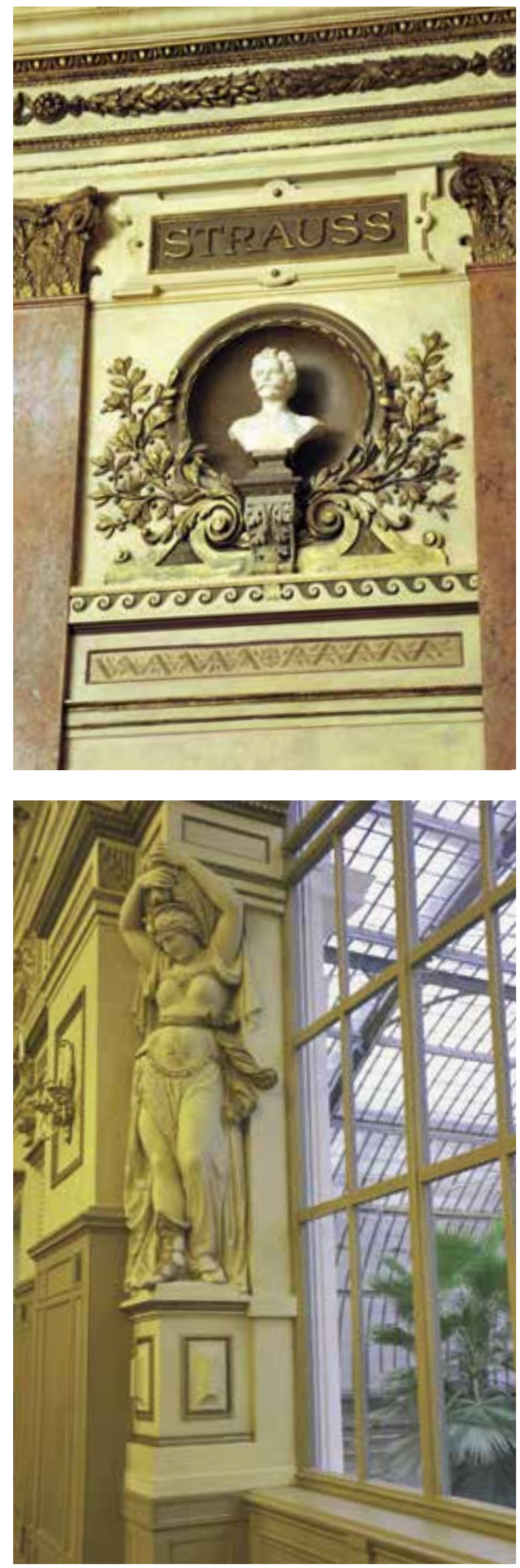